\title{
Assessing environmental risks associated with ultrafine coal wastes using laboratory-scale tests
}

\author{
A. K. B. Opitz ${ }^{1,2, a}$, J. L. Broadhurst ${ }^{1, b}$ and S. T. L. Harrison ${ }^{2, c}$ \\ ${ }^{1}$ Minerals to Metals Initiative, ${ }^{2}$ Centre for Bioprocessing Engineering Research (CeBER) \\ University of Cape Town, Rondebosch, Cape Town, South Africa. \\ aoptale001@myuct.ac.za, bJennifer.Broadhurst@uct.ac.za, cSue.Harrison@uct.ac.za
}

Keywords: Acid rock drainage, metal deportment, coal fines, waste characterisation

\begin{abstract}
Characterisation of the risk of acid rock drainage is typically achieved through the quantification of acid-generating and acid-consuming components present within a sample using initial laboratory-scale, chemical static tests. These static tests, however, consider ARD generation under chemical conditions and do not account for the role of micro-organisms. Their focus is exclusively on the net potential for acid generation, with no account of metal deportment or the relative rate of acid generation and consumption. The present study investigates the ARD potential of two ultrafine coal wastes samples using the standard static tests as well as the UCT biokinetic test to account for microbial ARD generation. The deportment of metal species under each test condition was also considered. The UCT biokinetic test results supported the static test classification, providing preliminary kinetic data on the ARD generation. Sequential chemical extraction tests allowed for differentiation of the host minerals according to their leaching potentials, providing supporting evidence for the deportment of metal species under the characterisation tests, thereby improving the knowledge base on which to classify coal wastes as benign or otherwise.
\end{abstract}

\section{Introduction}

Acid rock drainage (ARD) is formed from the oxidation of exposed sulphide minerals, especially pyrite, by oxygen in the presence of water. Under acidic conditions, ARD generation is exacerbated through the regeneration of ferric iron by iron-oxidising micro-organisms [1]. Sulfur-oxidising microorganisms oxidise associated sulfur-species, generating $\mathrm{H}^{+}$to aid further leaching [2]. Environmental effects of ARD result from generation of acidity, release of toxic metals, and generation of high soluble sulphate concentrations, with increased total salinity of surrounding environments [3]. Generation and associated effects last over many decades [4].

Accurate characterisation and prediction of ARD potentials is necessary to implement effective management strategies for pollution mitigation. To limit the characterisation time and cost, current characterisation protocols follow a sequential process [5]. Chemical static tests are performed to determine the overall net ARD potential, giving no information regarding the relative rates of acid generation and neutralisation, or its composition under disposal conditions. Subsequent kinetic prediction tests, performed to give information on rate of overall ARD generation, add significant cost to the characterisation process [6]. In the chemical static tests, the basis for ARD characterisation is the potential for net acid formation [7], with no attention given to the risks associated with metal deportment. The potential for metal deportment, with associated environmental degradation, exists in the absence of acid generation and is typically aggravated by the presence of acid. The current characterisation methods also fail to account for microbially-mediated ARD generation [8]. Recently, the UCT biokinetic test was developed to gain insight the potential for ARD generation under microbial conditions [9]. Further integration of the results obtained from this test with those obtained from the current static characterisation tests remains necessary. The present study was undertaken to investigate the environmental risks associated with two ultrafine coal waste samples in terms of the integration of static and microbial kinetic characterisation test results as well as metal deportment. 


\section{Experimental Setup}

Ultrafine Coal Waste. In the completion of this study, two ultrafine coal wastes (sample A \& B) were obtained from the Witbank and Waterberg coalfields in South Africa respectively. Analysis of the particle size distribution indicated a $\mathrm{D}_{80}$ of approximately $80 \mu \mathrm{m}$ and $68 \mu \mathrm{m}$ respectively.

Static ARD Characterisation Tests. Static ARD characterisation tests were performed on the coal waste samples. Standard acid-base accounting (ABA) [5], in conjunction with net acid generation (NAG) tests were performed in triplicate. To assess the ARD potential under microbially-mediated conditions, duplicate biokinetic weathering tests [9] were done using the UCT biokinetic protocol. Leachate solutions from the characterisation tests were collected for metal analysis.

Sequential Chemical Extraction Tests. Sequential chemical extractions tests [10] were performed on $1 \mathrm{~g}$ coal waste samples. The protocol used a 7-stage sequential reaction of the waste sample with leaching agents of increasing strength. Analysis of the solution from each leach stage for metal concentrations allowed partitioning of the host minerals according to their specific reactivity.

\section{Results \& Discussion}

The two ultrafine coal waste samples were classified by acid-base accounting (Table 1). Sample A was classified as potentially acid forming (PAF), with a total sulfur grade of $3.1 \%$ and a relatively low acid-neutralising capacity (ANC). Conversely, Sample B was classified as non-acid forming due to a higher ability to neutralise acid relative to the maximum potential for acid formation. The ABA classification of the two waste samples was supported by the NAG tests when plotted against the NAPP (Fig. 1). The $\mathrm{pH}$ of the solution following reaction with $15 \% \mathrm{H}_{2} \mathrm{O}_{2}$ indicated the PAF and NAF classifications for samples A and B respectively.

Table 1: ARD characterisation results for the ultrafine coal wastes following ABA test work.

\begin{tabular}{|l|c|c|c|c|c|}
\hline & $\begin{array}{c}\text { Sulfur } \\
\text { Grade [\%] }\end{array}$ & $\begin{array}{c}\mathrm{MPA} \\
{\left[\mathrm{kg} \mathrm{H}_{2} \mathrm{SO}_{4} / \text { ton }\right]}\end{array}$ & $\begin{array}{c}\mathrm{ANC} \\
{\left[\mathrm{kg} \mathrm{H}_{2} \mathrm{SO}_{4} / \text { ton }\right]}\end{array}$ & $\begin{array}{c}\mathrm{NAPP} \\
{\left[\mathrm{kg} \mathrm{H}_{2} \mathrm{SO}_{4} / \text { ton }\right]}\end{array}$ & $\begin{array}{c}\text { ABA Classification } \\
{\left[\mathrm{kg} \mathrm{H}_{2} \mathrm{SO}_{4} / \text { ton }\right]}\end{array}$ \\
\hline Sample A & 3.1 & 95 & 3.8 & 91 & $\mathrm{PAF}$ \\
\hline Sample B & 0.92 & 32 & 121 & -89 & $\mathrm{NAF}$ \\
\hline
\end{tabular}

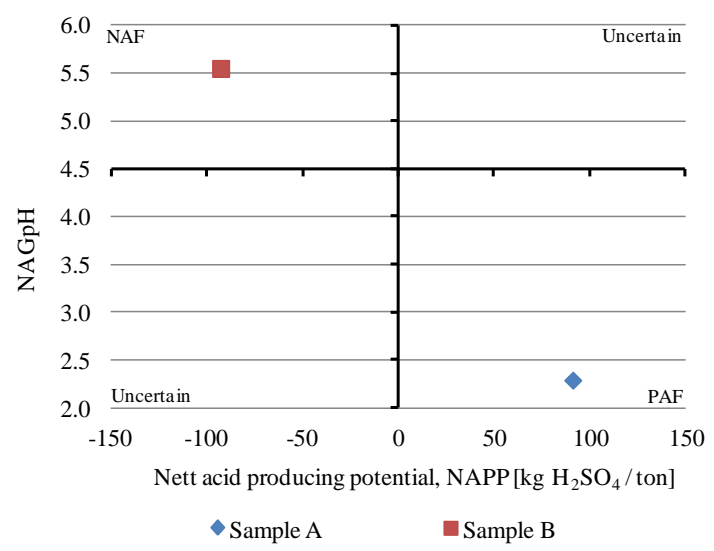

Fig. 1. Characterisation plot showing the ARD classification for Samples A and B as potentially acid forming (PAF) and non-acid forming (NAF) respectively.

The $\mathrm{pH}$ profiles of the biokinetic test results (Fig. 2A) supported the ABA and NAG classifications. The immediate, although small, rise in solution $\mathrm{pH}$ for waste sample A was indicative of the low ANC shown from the ABA test. The subsequent decrease in solution $\mathrm{pH}$ was due to microbiallymediated oxidation of sulfide minerals and intermediary sulfur species; these occurred at a slower relative rate to the acid neutralising reactions. Microbial ferrous iron oxidation to sustain ferric leaching was also demonstrated by the high redox potential shown in Fig. 2B. Conversely, the sharp rise in $\mathrm{pH}$ observed for waste sample $\mathrm{B}$ was indicative of the higher neutralising potential of this sample relative to its potential for acid formation. The $\mathrm{pH}$ remained above $\mathrm{pH} 7.0$ for the duration of this test, inhibiting microbial ferrous iron oxidation as well as chemical ferric oxidation of the 
sulphide minerals through removal of $\mathrm{Fe}^{3+}$ by precipitation. The relative absence of soluble ferric iron was suggested by the low redox potential values (Fig. 2B).
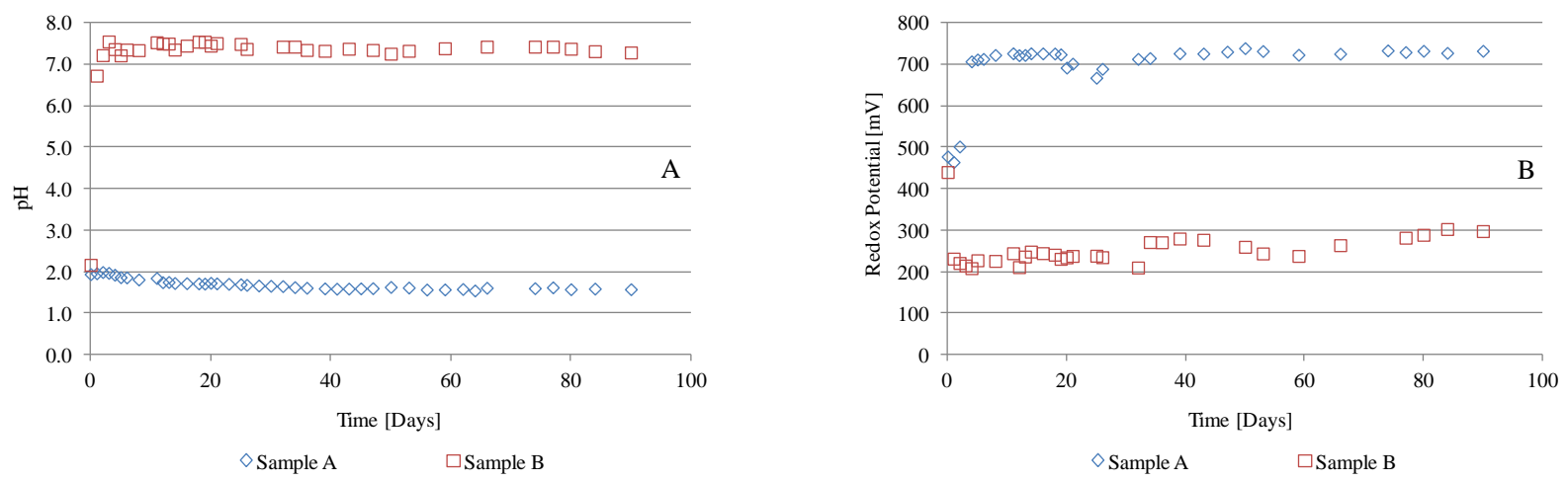

Fig. 2: pH (A) and redox potential (B) profiles from biokinetic tests performed on the coal waste samples.

Metal mobility under ANC conditions was due to acid dissolution of the mineral phases, whereas mobility under the NAG and biokinetic tests was due mineral oxidation, with acid dissolution resulting from the formation of acidity (Fig. 3). The low extractions of $\mathrm{Al}$ and $\mathrm{K}$ are consistent with these metal ions being associated with slow-reacting silicate mineral phases. For sample A, the difference in experimental duration resulted in a slight increase in the aluminium concentration of the biokinetic test as compared to the ANC and NAG tests. For sample B, the differences in aluminium and potassium concentrations between the static and biokinetic tests were due to precipitation of these species above $\mathrm{pH} 3.5$ [11].
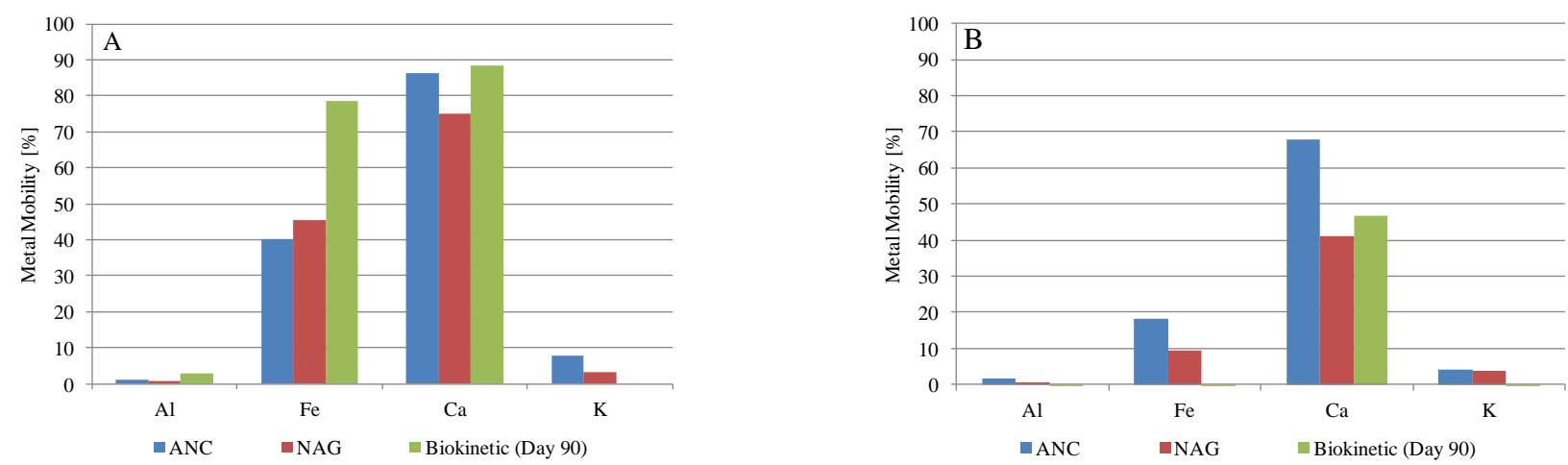

Fig. 3: Metal deportment of $\mathrm{Al}, \mathrm{Fe}, \mathrm{Ca}$, and $\mathrm{K}$ major metal species under $\mathrm{ANC}, \mathrm{NAG}$ and biokinetic test methods for waste sample A (A) and sample B (B).

For sample A, the differences in deportment of iron in the ANC and biokinetic tests resulted from the presence of iron-bearing mineral phases solubilised through oxidation only. This was not observed during NAG tests due to limited hydrogen peroxide availability, as suggested by the similarity in metal concentrations in the ANC and NAG test solutions. This limitation may have been due to oxidation of the organic sulfur species present within the coal samples (data not shown). The similar calcium concentrations were due to the acidity of the characterisation tests, possibly due to the presence of calcium oxide within South African coals [12]. The elevated $\mathrm{pH}$ conditions during NAG and biokinetic tests on sample B resulted in the precipitation of the aluminium and iron species within these tests relative to the ANC leachate. The elevated $\mathrm{pH}$ conditions were due to the presence of calcium-bearing, acid-consuming minerals as suggested by the calcium concentrations under all tests conditions.

The sequential chemical extraction results indicate the reactivity of the mineral phases associated with the specific metal species, hence the conditions for metal deportment. The low aluminium deportment was supported by the partitioning of the majority of aluminium host minerals within the residual mineral phase, with extraction of minerals from this phase occurring under aqua regia digestion only. The use of potassium chloride within the extraction protocol did not allow for reactive partitioning of the potassium-bearing mineral phases. 
For sample A, iron deportment under ANC conditions corresponded to the mineral phases which reported to the water soluble, exchangeable and carbonate reactive stages. The deportment of approximately $80 \%$ of the iron species under biokinetic test conditions supported the results of the sequential chemical extraction, corresponding to the first six sequential chemical extraction stages. This added credence to the suggestion that a lack of hydrogen peroxide reactant during the NAG test led to incongruent oxidation of the iron-bearing mineral phases.

Although the sequential extraction results indicate the potential for approximately 50\% iron deportment under acidic, oxidative conditions, the elevated $\mathrm{pH}$ conditions within the NAG and biokinetic test resulted in precipitation of the iron species. The observed deportment of calcium under ANC conditions correlated to solubilisation of the calcium associated with minerals present in the water-soluble, exchangeable and carbonate mineral phases.
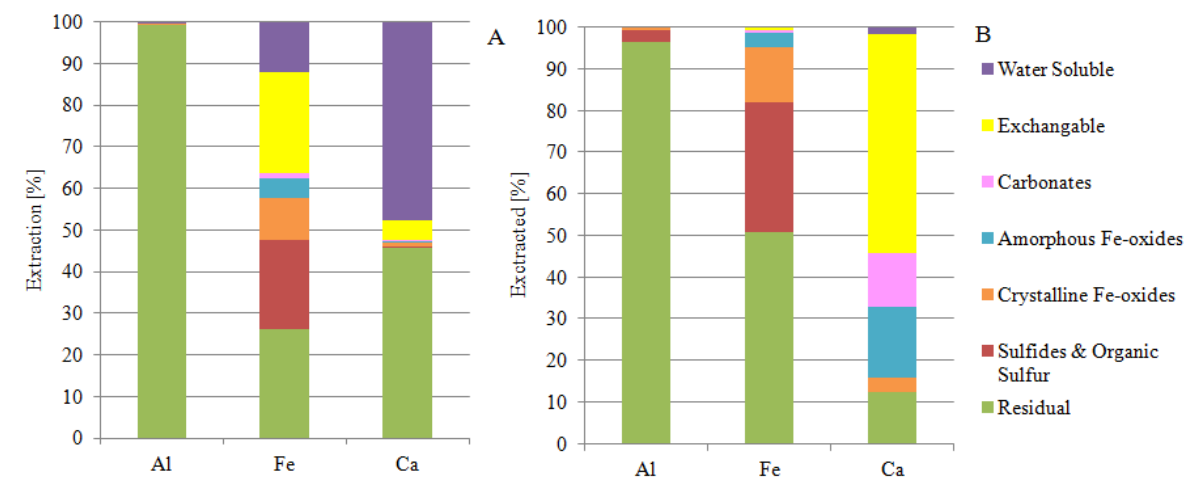

Fig. 4: Sequential chemical extraction results for coal waste samples A (A) and B (B) showing reactive mineral partitioning for the major metals

\section{Conclusions}

The results from the biokinetic characterisation test supported the classification of the waste samples provided by the chemical static tests. Decoupling of the relative rates of the acid-neutralisation and acid-formation was achieved for the acid-generating sample using analysis of the solution $\mathrm{pH}$ with time. The standard NAG methodology does not account for acid generation from non-sulfide mineral sources, with the hydrogen peroxide availability forming a limiting factor. Analysis of the soluble metal concentrations provided information regarding metal deportment under the different test conditions, supported by results from provided by the SCE tests. Partitioning of mineral phases according to their reactivity allows for prediction of metal deportment under disposal conditions.

\section{References}

[1] D.B. Johnson and K.B. Hallberg: Res. Microbiol. Vol. 154 (2003), p. 466.

[2] A. Schippers and W. Sand: Appl. Environ. Microb. Vol. 65 (1999), p. 319.

[3] D.K. Nordstrom and C.N. Alpers: Rev. Econ. Geol. Vol. 6A (1999), p. 133.

[4] S.D. Miller, W.S. Stewart, Y. Rusdinar, R.E. Schumann, J.M. Ciccarelli, J. Li and R.S. Smart: Sci. Total. Environ. Vol. 408 (1999), p. 2129.

[5] R. Smart, B. Skinner, G. Levay, A. Gerson, J. Thomas, H. Sobieraj, R. Schumann, C. Weisener, P. Weber, S. Miller and W. Stewart, ARD Test Handbook, 2002, Ian Wark Research Institute: Australia.

[6] M.F. Lengke, A. Davis and C. Bucknam: Mine Water Environ. Vol. 29 (2010), p. 29.

[7] W.A. Stewart, S.D. Miller and R. Smart, in: Proceedings of the $7^{\text {th }}$ International Conference on Acid Rock Drainage (ICARD), edited by R.I. Barnhisel, St Louis, Missouri, USA (2006).

[8] W.A. Price. In: Environmental Aspects of Mine Wastes, edited by J.L. Jambor, D.W. Blowes and A.I.M. Richie, Vancouver, Canada (2003).

[9] A.H. Hesketh, J.L. Broadhurst, C.G. Bryan, R.P. van Hille and S.T. Harrison: Miner. Eng. Vol. 104 (2010), p. 459.

[10] J.L. Broadhurst, W.J. Malukele and H. von Blottnitz, in: Proceedings of the $8^{\text {th }}$ International Conference on Acid Rock Drainage (ICARD), Skelefteå, Sweden (2009).

[11] C. Carbone, E. Dinelli, P. Marescotti, G. Gasparotto and G. Lucchetti: Geochem. Explor. Vol. 132 (2013), p. 188.

[12] K.L. Pinetown, C.R. Ward and W.A. van de Westerhuizen: Int. J. Coal. Geol. Vol 70 (2007), p. 166. 